\title{
Efecto del tratamiento higro-térmico en las características acústicas de la madera de Quercus scytophylla Liebm.
}

\section{Effect of hygro-thermal treatment in the acoustic characteristics of the wood of Quercus scytophylla Liebm.}

\author{
Javier Ramón Sotomayor-Castellanos’, "Germán Suárez-Béjarly Joel Benancio Olguín-Cerón'
}

1 Facultad de Ingeniería en Tecnología de la Madera.
Universidad Michoacana de San Nicolás de Hidalgo.

Universidad Michoa
Michoacán, México.

\section{RESUMEN}

La madera del género Quercus presenta un potencial importante como material para la elaboración de productos de madera con alto valor agregado. Con el objetivo de verificar si un tratamiento higro-térmico aplicado a la madera de Quercus scytophylla, modifica de manera significativa su módulo de elasticidad y sus propiedades de amortiguamiento, se estudió el efecto del tratamiento en sus características acústicas. Se ensayaron 30 probetas sin tratamiento y 30 con tratamiento en vibraciones transversales y se calcularon los parámetros densidad, contenido de humedad, frecuencia natural, módulo de elasticidad, módulo específico, decremento logarítmico, coeficiente de amortiguamiento y coeficiente acústico. Análisis estadísticos demostraron que la frecuencia natural es un buen predictor de los módulos de elasticidad y que el tratamiento higro-térmico modificó significativamente la densidad y el decremento logarítmico.

PAlabras Clave: coeficiente acústico, coeficiente de amortiguamiento, decremento logarítmico, frecuencia natural, módulo de elasticidad, vibraciones transversales

\section{ABSTRACT}

The influence of hygro-thermal treatment in the acoustic characteristics of Quercus scytophylla wood was studied. 30 specimens without treatment and 30 with treatment were tested in bending vibration (free-free supported). Density, moisture content, natural frequency, modulus of elasticity in bending vibration, specific modulus, logarithmic decrement, damping coefficient and acoustic coefficient were computed. Statistical analyses demonstrated that natural frequency is a good predictor of the modulus of elasticity and that hygrothermal treatment modified significantly the density and logarithmic decrement.

KEY WORDS: acoustic coefficient, damping coefficient, logarithmic decrement, natural frequency, modulus of elasticity, bending vibration.

\section{INTRODUCCIÓN}

Las pruebas de vibraciones transversales han confirmado su utilidad para medir la frecuencia natural de una pieza de madera. Ponderando esta propiedad con la densidad y otros parámetros derivados de sus dimensiones, este método no destructivo es empleado para predecir el módulo de elasticidad dinámico y el coeficiente de amortiguamiento de la madera (Kawamoto y Williams, 2002).

El módulo de elasticidad dinámico de la madera es un indicador de su resistencia a la deformación elástica y es uno de los parámetros de ingeniería más importantes para el diseño de productos y el cálculo de estructuras de 
madera. Entre otras aplicaciones, este módulo se emplea para dimensionar elementos estructurales que satisfagan requisitos de calidad y de seguridad estructural. El coeficiente de amortiguamiento de la madera es un índice de su capacidad para disipar energía y es un parámetro importante en el cálculo y diseño de un elemento estructural o de un producto de madera que están sometidos a vibraciones (Tamarit y López, 2007).

Un buen diseño de estructuras de madera puede contribuir a mejorar el ambiente sonoro en construcciones, gracias a las propiedades acústicas del material. Entre otros indicadores de calidad de los materiales de construcción, el módulo elástico específico y el coeficiente acústico, son indicadores de la calidad de la madera para usos específicos donde se recomienda una alta resistencia en relación con su densidad. Además, son también indicadores para su empleo en productos o en aplicaciones donde el comportamiento acústico es relevante (Ashby, 2010). Las propiedades de la madera relacionadas con su caracterización acústica son importantes también para su valoración como materia prima para la elaboración de muebles usados como amplificadores de sonido y cajas de resonancia musicales (Bucur, 2006).

La caracterización mecánica-acústica de la madera ha sido posible gracias a la aplicación de métodos de evaluación de carácter no destructivo en el estudio de especies con vocación acústica y constructiva. Particularmente, las técnicas que utilizan ondas y vibraciones mecánicas para estimar su módulo de elasticidad, están documentadas recientemente, entre otros, por Sandoz et al. (2000), Pellerin y Ross (2002), Bucur (2006) y Ortiz et al. (2009).

Respecto a las características módulo de elasticidad dinámico y coeficiente de amortiguamiento de la madera, está documentado que estas varían de acuerdo con la composición química de la especie (Minato et al., 2010), según el tratamiento químico a que sea sometida la madera (Brémaud et al., 2010) y dependen también de las características anatómicas de cada especie (Brancheriau et al., 2006a y 2006b; Brémaud et al., 2011; Roohnia et al., 2011a).

En la bibliografía especializada del país, se encuentran datos de características elásticas de maderas mexica- nas determinadas en condiciones de carga estáticas (Tamarit y López, 2007; Silva-Guzmán et al., 2010). Con todo, es notoria la escasez de datos derivados de pruebas mecánicas que puedan aplicarse en el estudio del comportamiento de productos funcionando en condiciones dinámicas, o para fines de diseño sísmico de estructuras.

En México existen publicaciones que proponen maderas mexicanas para su estudio y promoción en aplicaciones acústicas. Entre otros autores se pueden citar: Guridi y García (1997); Barajas y León (1984); Orduña y Quintanar (1992); Quintanar et al. (1998); Gutiérrez y Dorantes (2007); De la Paz et al. (2002); Tamarit y López (2007). Estos trabajos se orientan principalmente a la caracterización de la estructura anatómica y de los posibles usos de la madera en instrumentos musicales. Con excepción de Sotomayor-Castellanos et al. (2010 y 2013), Sotomayor-Castellanos y Suárez-Béjar (2013) y Sotomayor-Castellanos y Olguín-Cerón (2014), no se encontró información acerca de características acústicas y/o índices de calidad de especies la madera del género Quercus que habitan en el País.

La madera del género Quercus, es endémica del Estado de Michoacán y presenta un potencial importante como material para la elaboración de productos de madera (Bello y Labat, 1987). De acuerdo con sus características anatómicas, De la Paz Pérez et al. (2006) recomiendan la madera de Q. scytophylla para la elaboración de productos de alta calidad que pongan de manifiesto sus características estéticas, entre otros: ebanistería, chapa, pisos, juguetes, muebles y partes de instrumentos musicales. Estos autores indican esta madera tiene menos problemas para el aserrío y el secado, comparativamente con otras especies de Quercus.

\section{Comportamiento higro-térmico}

La resistencia mecánica de la madera no es afectada de manera permanente si esta es expuesta por períodos cortos a temperaturas menores a $100{ }^{\circ} \mathrm{C}$. Sin embargo la resistencia puede disminuir de manera permanente si la madera se expone a una temperatura mayor de $65^{\circ} \mathrm{C}$, durante períodos de tiempo prolongado. Los factores principales en la modificación de la resistencia de la madera son la tempera- 
tura del tratamiento, el medio de transferencia de calor, el contenido de humedad de la madera, la especie y el volumen de la madera a tratar (Oltean et al., 2007). Este fenómeno se observa para temperaturas superiores a $100{ }^{\circ} \mathrm{C}$. En tratamientos térmicos comprendidos en el intervalo que va de $0{ }^{\circ} \mathrm{C}$ a $100{ }^{\circ} \mathrm{C}$, la madera sólida no presenta una influencia permanente en lo que respecta a sus propiedades mecánicas (Estevez y Pereira, 2009).

Brémaud et al. (2011) compilaron datos bibliográficos del módulo de elasticidad en vibraciones transversales $\mathrm{y}$ del coeficiente de amortiguamiento $(\tan \delta)$ en pruebas con condiciones de $20{ }^{\circ} \mathrm{C}$ a $25{ }^{\circ} \mathrm{C}$ y $55 \%$ a $65 \%$ de humedad relativa, en un intervalo de frecuencias de $50 \mathrm{~Hz}$ a $2000 \mathrm{~Hz}$, para maderas de especies angiospermas con densidad $(\rho)$ de $660 \mathrm{~kg} / \mathrm{m}^{3}$, módulo de elasticidad $\left(E_{v t}\right)$ de $13500 \mathrm{MPa}$ y coeficiente de amortiguamiento $(\tan \delta)$ de $8,1 \times 10^{-3}$ y para maderas gimnospermas con densidad de $440 \mathrm{~kg} / \mathrm{m}^{3}, E_{v t}=11040 \mathrm{MPa}$ y $\tan \delta=7,1 \times 10^{-3}$. Obataya et al. (1998) determinaron en vibraciones transversales, para madera de Picea sitchensis $\left(\rho=448 \mathrm{~kg} / \mathrm{m}^{3}\right.$ y $\mathrm{CH}=$ $12,7 \%$ ), módulos de elasticidad en promedio de 12950 MPa y valores promedio de $\tan \delta$ de 7,1 x $10^{-3}$.

Brancheriau et al. (2010) determinaron para madera de Dalbergia sp., $\left(\rho=960 \mathrm{~kg} / \mathrm{m}^{3}\right.$ y $\mathrm{CH}=$ promedio de $15 \%$, valores promedio de $\tan \delta$ de $3,8 \times 10^{-3}$ en ensayos de vibraciones transversales con frecuencias de entre 400 $\mathrm{Hz}$ y $1000 \mathrm{~Hz}$. Sedik et al. (2010) determinaron para seis especies angiospermas: Endospermum diadenum ( $\rho=$ $\left.350 \mathrm{~kg} / \mathrm{m}^{3}\right)$, Cratoxylum arborecens $\left(\rho=410 \mathrm{~kg} / \mathrm{m}^{3}\right)$, Dyera polyphylla $\left(\rho=440 \mathrm{~kg} / \mathrm{m}^{3}\right)$, Macaranga gigantea $(\rho$ $\left.=240 \mathrm{~kg} / \mathrm{m}^{3}\right)$, Commersonia bartramia $\left(\rho=320 \mathrm{~kg} / \mathrm{m}^{3}\right) \mathrm{y}$ Alstonia pneumatophora $\left(\rho=370 \mathrm{~kg} / \mathrm{m}^{3}\right)$ un módulo en vibraciones transversales libre-libre promedio de 22270 $\mathrm{MPa}$, todas las maderas con $\mathrm{CH}$ promedio de $12 \%$. Zhang et al. (2011) calcularon para tres especies angiospermas: Simarouba amara $\left(\rho=391 \mathrm{~kg} / \mathrm{m}^{3}\right)$, Carapa procera $(\rho=$ $\left.655 \mathrm{~kg} / \mathrm{m}^{3}\right)$ y Symphonia globulifera $\left(\rho=709 \mathrm{~kg} / \mathrm{m}^{3}\right)$, con $\mathrm{CH}$ promedio de $10,7 \%$, el módulo específico en vibraciones transversales $E_{\rho h}$ en un intervalo de $16000 \mathrm{MPa}$ a 35 $000 \mathrm{MPa}$ y un coeficiente de amortiguamiento $(\tan \delta)$ en un intervalo de $5 \times 10^{-3}$ a $9,5 \times 10^{-3}$.
Sotomayor-Castellanos et al. (2010) calcularon para madera de Q. scytophylla, $\left(\rho=813 \mathrm{~kg} / \mathrm{m}^{3}\right.$ y $\left.\mathrm{CH}=10,7 \%\right)$ los módulos de elasticidad por ultrasonido, el índice material y el factor de calidad, para las direcciones radial y tangencial y longitudinal. Sotomayor-Castellanos et al. (2013) determinaron para maderas de Quercus spp. ( $\rho=$ $898 \mathrm{~kg} / \mathrm{m}^{3}$ y $\mathrm{CH}=10 \%$, los módulos de elasticidad en la dirección longitudinal por ultrasonido, ondas de esfuerzo y el módulo de elasticidad en vibraciones transversales $\left(E_{v t}\right.$ = $17600 \mathrm{MPa}$ ). Además, calcularon los índices de calidad correspondientes a estos tipos de solicitación. SotomayorCastellanos y Suárez-Béjar (2013) establecieron para madera del género Quercus, $\left(\rho=734 \mathrm{~kg} / \mathrm{m}^{3}\right.$ y $\mathrm{CH}=$ $13,17 \%$, la frecuencia natural $(901 \mathrm{~Hz})$, el módulo de elasticidad (11 805), el módulo específico $(22,26)$ y el coeficiente de amortiguamiento $\left(5,45 \times 10^{-3}\right)$ y el coeficiente acústico $(7,67)$, determinados en pruebas de vibraciones transversales. Sotomayor-Castellanos y Olguín-Cerón (2014) realizaron pruebas de ondas de esfuerzo en la dirección longitudinal en probetas de madera de Q. scytophylla, con y sin tratamiento de plastificado, y determinaron módulos de elasticidad de $11515 \mathrm{MPa}$ y $11385 \mathrm{MPa}$, respectivamente $\left(\rho=744 \mathrm{~kg} / \mathrm{m}^{3}\right.$ y $788 \mathrm{~kg} / \mathrm{m}^{3}$ y CH promedio $=11,96 \%$ ).

La frecuencia natural, la densidad, el módulo de elasticidad y el coeficiente de amortiguamiento de la madera, son buenos descriptores de sus características acústicas (Aramaki, 2007). Por ejemplo, una madera con un módulo de elasticidad especifico y un coeficiente acústico altos, combinados con propiedades de amortiguamiento bajas, se puede valorar como una especie con buenas características acústicas y con vacación para su empleo en la fabricación de componentes de aparatos e instrumentos musicales (Shen, 2006). En cambio, una madera con bajo coeficiente de amortiguamiento denota una vocación en productos de madera que requieren de disipación de energía, por ejemplo en herramientas de trabajo y en artículos deportivos y en elementos constructivos donde son importantes las propiedades de aislamiento de vibraciones, por ejemplo muros, pisos y componentes estructurales para diseño sísmico de edificaciones con madera (Meyers, 1994). 
En México existe una gran biodiversidad de especies forestales, de tal forma, que la caracterización mecánica de la madera es necesaria caso por caso de cada especie. La madera de Q. scytophylla tiene un gran potencial para fabricar productos de alto valor agregado. En el mismo contexto, aún no se han desarrollado procedimientos normalizados para pruebas dinámicas empleando métodos de evaluación no destructivos.

\section{OBjetivo}

El objetivo de la investigación es verificar experimentalmente si un tratamiento higro-térmico aplicado a la madera de Q. scytophylla, modifica de manera significativa su módulo de elasticidad y sus propiedades de amortiguamiento determinados en vibraciones transversales.

\section{MATERIALES Y MÉTODOS}

\section{Materiales}

El material experimental consistió en madera de la especie Quercus scytophylla Liebm., proveniente de un árbol colectado en el municipio de Morelia, Michoacán. La especie fue identificada por personal de la empresa concesionada para cosechar arbolado en esta región, de acuerdo con la ficha técnica de Quercus scytophylla Liebm., del Manual de la Biodiversidad de encinos michoacanos (Arizaga et al., 2009). Se recortaron 2 trozas de 1,1 m de largo y de $45 \mathrm{~cm}$ de diámetro, de las cuales se aserraron tablones de corte radial, de $5 \mathrm{~cm}$ por $12,5 \mathrm{~cm}$ de sección transversal. De los tablones, se preparó un primer grupo de 30 probetas, identificado como probetas sin tratamiento (PST). Además, se elaboró un segundo grupo de 30 probetas, identificadas como probetas con tratamiento (PCT). Este material pertenece al lote de madera investigado por Olguín-Cerón y Sotomayor-Castellanos (2013). Las dimensiones de las probetas fueron $32 \mathrm{~cm}$ en la dirección longitudinal, $1,9 \mathrm{~cm}$ en la dirección radial y $1,9 \mathrm{~cm}$ en la dirección tangencial de la madera, siguiendo las recomendaciones de la norma ISO 3129:2012 (International Organization for Standardization, 2012).

\section{Tratamiento higro-térmico}

El tratamiento higro-térmico de los listones destinados para las probetas con tratamiento, consistió primeramente en el hidratado de la madera. Los listones se mantuvieron inmersos en agua, es decir, en estado húmedo y saturado durante 30 días, con el objeto de uniformizar el contenido de humedad de la madera. Posteriormente, los listones se trataron en el dispositivo de suavizado con una secuela de temperatura y contenido de humedad de la madera usando tres períodos de temperatura: el primero de 15 minutos, necesario para generar vapor al calentar 5 litros de agua en el generador de vapor. El segundo período de 30 minutos permite elevar la temperatura ambiente al interior de la cámara desde $23^{\circ} \mathrm{C}$, hasta la temperatura de suavizado de $98^{\circ} \mathrm{C}$. Al mismo tiempo, la temperatura interna de la madera alcanza $92{ }^{\circ} \mathrm{C}$. El tercer período de 50 minutos de duración mantiene estables las temperaturas al interior de la cámara de suavizado y al interior de la madera. Los detalles del proceso pueden ser consultados en OlguínCerón y Sotomayor-Castellanos (2013).

Una vez terminado el tratamiento de los listones para el grupo de probetas con tratamiento, el contenido de humedad de los dos grupos de listones se uniformizó durante 36 horas en condiciones de laboratorio con temperatura igual a $23{ }^{\circ} \mathrm{C}$ y humedad relativa de $36 \%$, siguiendo los criterios de Olguín-Cerón y SotomayorCastellanos (2013). Posteriormente, todos los listones se colocaron durante 130 horas en una cámara de acondicionamiento con una temperatura de $20^{\circ} \mathrm{C}$ y una humedad relativa de $65 \%$, hasta alcanzar un peso constante y contenido de humedad promedio de $11,8 \%$. Finalmente, se recortaron las probetas.

\section{Densidad y contenido de humedad}

La densidad de la madera $\left(\rho_{b}\right)$ se calculó con la relación peso/volumen al momento del ensayo de cada probeta con la fórmula (Bodig y Jayne, 1982):

$$
\rho_{h}=\frac{\rho_{h}}{V_{b}}
$$


Donde:

$\rho_{h} \quad=$ densidad de la madera al momento del ensayo $\left(\mathrm{kg} / \mathrm{m}^{3}\right)$

$p_{h} \quad=$ peso de la probeta al momento del ensayo $(\mathrm{kg})$

$V_{b} \quad=$ volumen de la probeta al momento del ensayo $\left(\mathrm{m}^{3}\right)$.

El contenido de humedad $(\mathrm{CH})$ de la madera se calculó como la proporción del peso del agua contenida por la probeta al momento del ensayo y el peso de la probeta en su estado seco, con la fórmula (Bodig y Jayne, 1982):

$$
\mathrm{CH}=\frac{\rho_{b}-\rho_{s}}{\rho_{s}}(100)
$$

Donde:

$\mathrm{CH}=$ contenido de humedad de la madera $(\%)$

$p_{h} \quad=$ peso de la probeta al momento del ensayo $(\mathrm{kg})$

$p_{s} \quad=$ peso de la probeta en estado seco $(\mathrm{kg})$.

\section{Pruebas de vibraciones transversales}

Las pruebas de vibraciones consistieron en poner en movimiento a la probeta en condición de apoyo libre- libre y medir la frecuencia natural y el decremento logarítmico de las vibraciones. La condición libre-libre se logró sujetando la probeta a dos apoyos elásticos considerados con rigidez insignificante y ambos colocados en los nodos del primer modo de vibración de la probeta. La configuración de las pruebas se presenta en la figura 1. Las vibraciones se lograron por medio de un impacto elástico $(P)$ en dirección transversal a la dirección longitudinal $(L)$ de la probeta empleando un martillo РСB Piezotronics ${ }^{\circledR}$, modelo 086B05 SN 4160. Para medir el desplazamiento de la probeta en la dirección transversal, se colocó un acelerómetro marca РСВ Piezotronics®, modelo 353B04 (Peso $=10,5 \mathrm{~g})$ en un extremo de cada pieza de madera, adherido con una cera adhesiva (Petro Wax 080A109, РCB Piezotronics®).

Una vez puesta la probeta en vibración, se midió la frecuencia natural a partir del diagrama del dominio de frecuencias obtenido con un algoritmo de la transformada rápida de Fourier. Al mismo tiempo, se calculó el decremento logarítmico a partir de la señal del amortiguamiento de las vibraciones. Siguiendo el protocolo

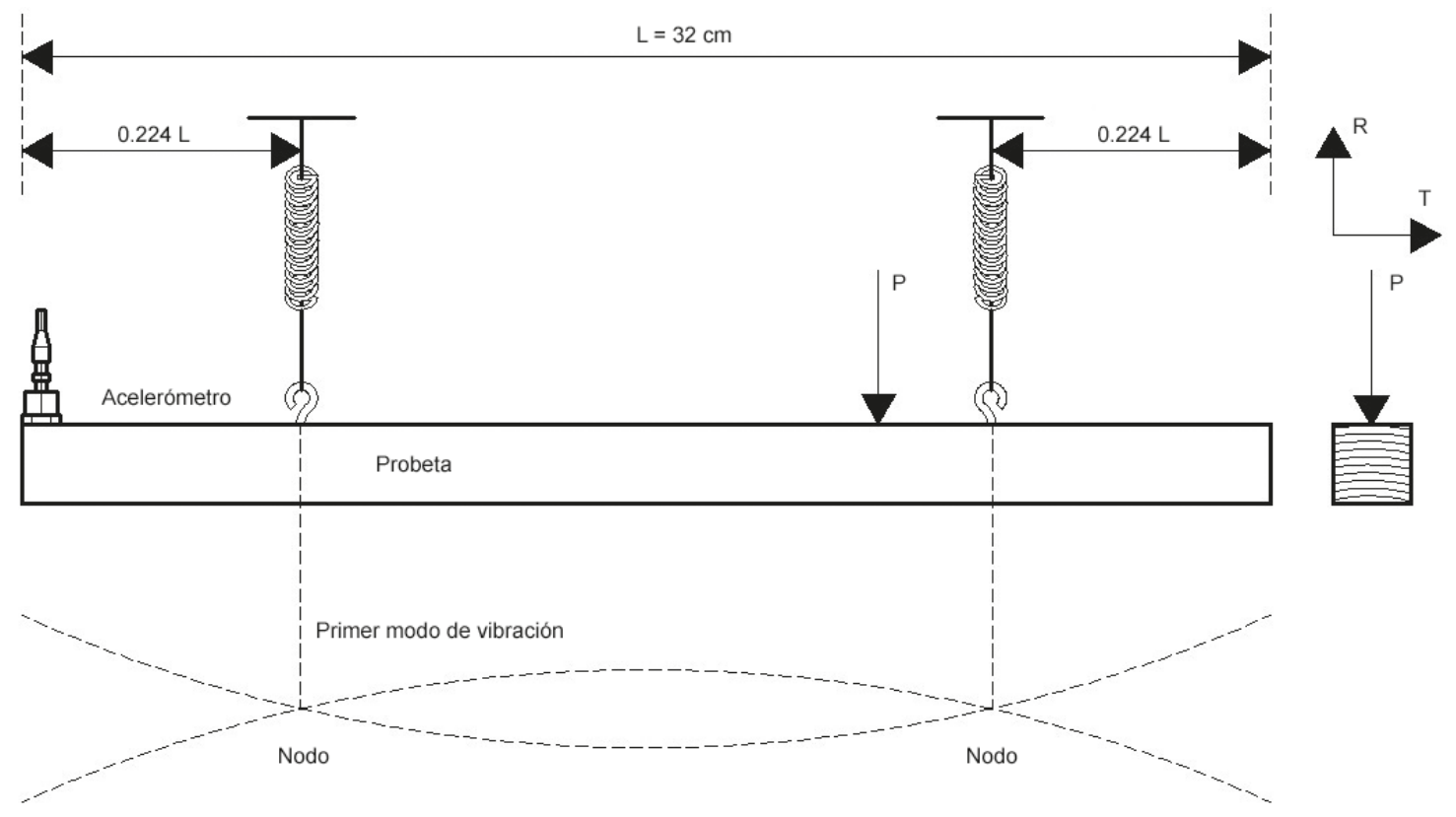

Figura 1. Configuración de las pruebas de vibraciones libre-libre. 
propuesto por Sotomayor-Castellanos y Suárez-Béjar (2013), la frecuencia natural y el decremento logarítmico fueron calculados por un analizador de señales dinámicas marca Brüel and Kjær@ ${ }^{\circledR}$, modelo 986A0186, provisto con un programa de adquisición y tratamiento de datos marca Brüel and Kjær@, modelo DSA-104. La intensidad del impacto y la amplitud de las vibraciones fueron reguladas con ayuda del sistema de adquisición y tratamiento de datos. Un diagrama de la señal del amortiguamiento de las vibraciones es presentado en la figura 2 y un diagrama de una frecuencia en el dominio de frecuencias es presentado en la figura 3.

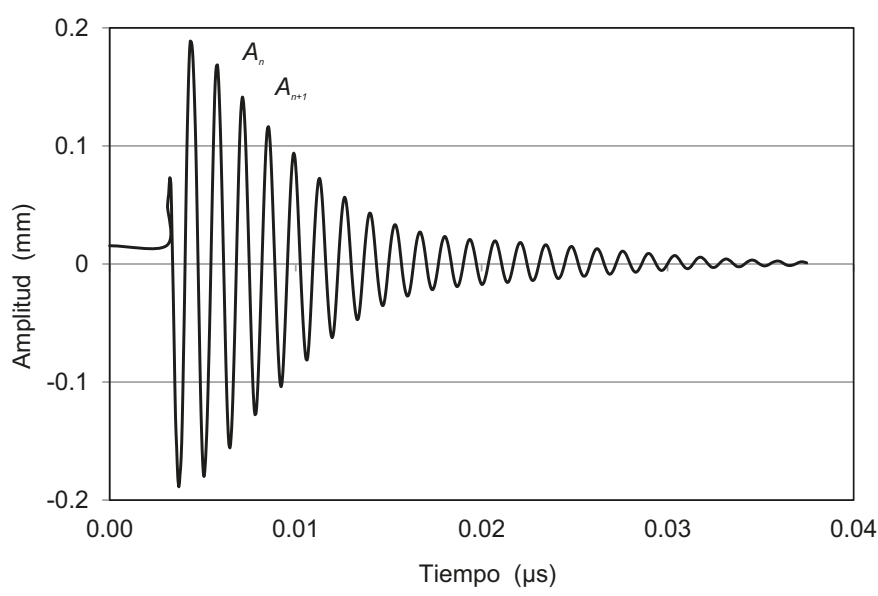

Figura 2. Señal del amortiguamiento de las vibraciones.

$A_{n}=$ Amplitud de la vibración en el ciclo $n ; A_{n+1}=$ Amplitud de la vibración en el ciclo $\mathrm{n}+\mathrm{l}$.



Figura 3. Diagrama del dominio de frecuencias.
El módulo de elasticidad en vibraciones se calculó con la fórmula (Pellerin y Ross, 2002):

$$
E_{v t}=\frac{f_{n}^{2} m L^{3}}{12,65 I}
$$

Donde:

$E_{v t} \quad=$ módulo de elasticidad de la madera $(\mathrm{Pa})$

$f_{n} \quad=$ frecuencia natural de la probeta $(\mathrm{Hz})$

$m \quad=$ masa de la probeta al momento del ensayo $(\mathrm{kg})$

$L \quad=$ longitud de la probeta $(\mathrm{m})$

I = momento de inercia de la sección transversal de la probeta $\left(\mathrm{m}^{4}\right)$.

El módulo específico se calculó con la fórmula (Roohnia et al. 2011a):

$$
E_{\rho h}=\frac{E_{v t}}{\rho_{h}}
$$

Donde:

$E_{\rho h} \quad=$ módulo específico $\left(\mathrm{m}^{2} / \mathrm{s}^{2}\right)$

$E_{v t} \quad=$ módulo de elasticidad $(\mathrm{Pa})$

$\rho_{h} \quad=$ densidad de la madera al momento del ensayo $\left(\mathrm{kg} / \mathrm{m}^{3}\right)$.

El coeficiente acústico se calculó con la fórmula (Wegst, 2006):

$$
K=\sqrt{\frac{E_{v t}}{\rho_{b}{ }^{3}}}
$$

Donde:

$K=$ coeficiente acústico $\left(\mathrm{m}^{4} / \mathrm{s} \mathrm{kg}\right)$

$E_{v t} \quad=$ módulo de elasticidad $(\mathrm{Pa})$

$\rho_{b} \quad=$ densidad de la madera al momento del ensayo $\left(\mathrm{kg} / \mathrm{m}^{3}\right)$.

El decremento logarítmico se calculó con la fórmula (Raichel, 2006):

$$
\delta=\ln \frac{A_{n}}{A_{n+1}}
$$


Donde:

$\delta \quad=$ decremento logarítmico

$A_{n} \quad=$ amplitud de la vibración en el ciclo $\mathrm{n}(\mathrm{m})$

$A_{n+1} \quad=$ amplitud de la vibración en el ciclo $\mathrm{n}+1(\mathrm{~m})$.

El coeficiente de amortiguamiento se calculó con la fórmula (Brémaud et al., 2010):

$$
\tan \delta=\tan (\delta)
$$

Donde:

$\delta \quad=$ decremento logarítmico

$\tan (\delta)=$ coeficiente de amortiguamiento.

\section{Análisis estadístico}

Se diseñaron tres análisis siguiendo las recomendaciones de Gutiérrez y de la Vara (2012). Los cálculos estadísticos fueron realizados con el programa Statgraphics ${ }^{\circledR}$. El primer análisis consistió en calcular los coeficientes de determinación $\left(R^{2}\right)$ para cada par de las cinco variables: densidad $\left(\rho_{h}\right)$, frecuencia natural $\left(f_{n}\right)$, módulo de elasticidad en vibraciones transversales $\left(E_{v t}\right)$, módulo específico $\left(E_{\rho h}\right)$ y decremento logarítmico $(\delta)$, correspondientes a cada uno de los dos grupos de probetas: probetas sin tratamiento (PST) y probetas con tratamiento (PCT). Para las probetas sin tratamiento y con tratamiento, se efectuaron pruebas con 30 réplicas en los dos casos.

El segundo análisis comparó las medias de los cinco parámetros: $\rho_{h}, f_{n}, E_{v t}, E_{\rho b}$ y $\delta$, como las variables de respuesta evaluadas para cada uno de los dos tipos de probetas: PST y PCT. El tercer análisis consistió en estudiar la influencia del peso del acelerómetro colocado en las probetas, sobre la medición de la frecuencia natural del sistema y su efecto en la magnitud del módulo de elasticidad $E_{v t}$. Para ello, se compararon la media de los valores de los módulos $E_{v t}$, determinados sin considerar el peso del acelerómetro, versus la media de los valores de los módulos $E_{v t}$, esta vez incorporando el peso de la probeta durante el ensayo.

Suponiendo una distribución normal con media cero $(\bar{x}=0)$ y varianza constante $\left(\sigma^{2}\right)$ e independientes entre sí, para cada prueba de diferencia de medias se verificó la hipótesis nula $H_{0}: \bar{x}_{1}-\bar{x}_{2}=0$, y se contrastó con la hipótesis alterna $H_{A}: \bar{x}_{1}-\bar{x}_{2} \neq 0$. El método empleado para discriminar entre las medias fue una prueba de Fisher con una diferencia mínima significativa de $95 \%$.

\section{RESULTADOS Y DISCUSIÓN}

La tabla 1 presenta, para los dos grupos de probetas PST y PCT, los valores de media aritmética, desviación estándar y coeficiente de variación, para los parámetros densidad, contenido de humedad, frecuencia natural, módulo de elasticidad en vibraciones transversales, módulo específico, decremento logarítmico, coeficiente de amortiguamiento y coeficiente acústico.

\section{Densidad}

La densidad de la madera con tratamiento disminuyó en $1,3 \%$ con respecto a la madera sin tratamiento. Este fenómeno en la disminución de la densidad de la madera como resultado de un tratamiento de temperatura e hidratado ha sido observado por Korkut et al. (2010) para la madera de Quercus petraea $\left(\rho_{H}=710 \mathrm{~kg} / \mathrm{m}^{3}\right)$, con una disminución de la densidad de $1 \%$, para un tratamiento con temperatura de $120^{\circ} \mathrm{C}$, aplicada durante 2 horas, condiciones parecidas a las del tratamiento aplicado a la madera de $Q$. scytophylla.

Los valores mínimos y máximos de la densidad $\rho_{b}$ para las probetas sin tratamiento fueron respectivamente de $819 \mathrm{~kg} / \mathrm{m}^{3}$ y de $880 \mathrm{~kg} / \mathrm{m}^{3}$. Para las probetas con tratamiento fueron de $707 \mathrm{~kg} / \mathrm{m}^{3}$ y $899 \mathrm{~kg} / \mathrm{m}^{3}$. De manera comparativa; las magnitudes de la densidad básica $\left(\rho_{0}\right)$ determinadas para el mismo material experimental por Sotomayor-Castellanos et al. (2014) son respectivamente para probetas sin tratamiento $\left(\rho_{0}\right.$ mínima $=676 \mathrm{~kg} / \mathrm{m}^{3} ; \rho_{0}$ máxima $\left.=766 \mathrm{~kg} / \mathrm{m}^{3}\right)$ y con tratamiento $\left(\rho_{0}\right.$ mínima $=630$ $\mathrm{kg} / \mathrm{m}^{3} ; \rho_{0}$ máxima $\left.=786 \mathrm{~kg} / \mathrm{m}^{3}\right)$. La densidad calculada en esta investigación incluye el peso del agua del contenido de humedad de la madera al momento del ensayo. En cambio, la densidad básica está calculada con el peso de la madera en estado anhidro. 
TABla 1. Características acústicas de la madera de Quercus scytophylla.

\begin{tabular}{|c|c|c|c|c|}
\hline Parámetros (Símbolo) & Unidades & $\bar{x}$ & $\sigma$ & $C V$ \\
\hline \multicolumn{5}{|c|}{ Probetas sin Tratamiento (PST) } \\
\hline Densidad $\left(\rho_{H}\right)$ & $\left(\mathrm{kg} / \mathrm{m}^{3}\right)$ & 852 & 14,93 & 0,02 \\
\hline Contenido de humedad $(\mathrm{CH})$ & (\%) & 11,96 & 0,35 & 0,03 \\
\hline Frecuencia natural $\left(f_{n}\right)$ & $(\mathrm{Hz})$ & 747 & 41,57 & 0,06 \\
\hline Módulo de elasticidad $\left(E_{v t}\right)$ & (MPa) & 13922 & 1505 & 0,11 \\
\hline Módulo específico $\left(E_{\rho h}\right)$ & $\left(\mathrm{m}^{2} / \mathrm{s}^{2}\right)$ & 16,33 & 1,73 & 0,11 \\
\hline Decremento logarítmico $(\delta)$ & - & 0,2522 & 0,111 & 0,44 \\
\hline $\begin{array}{l}\text { Coeficiente de amortigua- } \\
\text { miento (tan } \delta \text { ) }\end{array}$ & $\times 10^{-3}$ & 4,40 & 1,93 & 0,44 \\
\hline Coeficiente acústico $(K)$ & $\left(\mathrm{m}^{4} / \mathrm{s} \mathrm{kg}\right)$ & 4,74 & 0,254 & 0,05 \\
\hline
\end{tabular}

$\bar{x}=$ Media aritmética; $\sigma$ = Desviación estándar; $C V$ = Coeficiente de variación.

\section{Influencia del acelerómetro en la frecuencia y en el módulo de elasticidad}

Durante las pruebas de flexión se posicionó un acelerómetro en uno de los extremos de cada probeta (Fig. 1), de tal forma, que su masa se adiciona a la de la probeta e influye en la medición de la frecuencia natural del sistema en vibración. En consecuencia, la magnitud del módulo de elasticidad calculado con la fórmula 3 es modificada. Si se modela la prueba de flexión transversal como un sistema en vibración con un grado de libertad, su frecuencia se define como (Meyers, 1994):

$$
f=\sqrt{\frac{k}{m}}
$$

Donde:

$$
\begin{array}{ll}
f & =\text { frecuencia }(\mathrm{Hz}) \\
k & =\operatorname{rigidez}(\mathrm{N} / \mathrm{m}) \\
m & =\operatorname{masa}(\mathrm{kg}) .
\end{array}
$$

En el caso del sistema en estudio, la rigidez $(k)$ está

\begin{tabular}{|c|c|c|c|c|}
\hline Parámetros (Símbolo) & Unidades & $\bar{x}$ & $\sigma$ & $C V$ \\
\hline \multicolumn{5}{|c|}{ Probetas con Tratamiento (PCT) } \\
\hline Densidad $\left(\rho_{H}\right)$ & $\left(\mathrm{kg} / \mathrm{m}^{3}\right)$ & 840 & 37,61 & 0,04 \\
\hline Contenido de humedad $(\mathrm{CH})$ & $(\%)$ & 11,70 & 0,95 & 0,08 \\
\hline Frecuencia natural $\left(f_{n}\right)$ & $(\mathrm{Hz})$ & 745 & 50,06 & 0,07 \\
\hline Módulo de elasticidad $\left(E_{v v}\right)$ & (MPa) & 13751 & 2146 & 0,16 \\
\hline Módulo específico $\left(E_{\rho h}\right)$ & $\left(\mathrm{m}^{2} / \mathrm{s}^{2}\right)$ & 16,34 & 2,27 & 0,14 \\
\hline Decremento logarítmico $(\delta)$ & - & 0,3610 & 0,187 & 0,52 \\
\hline $\begin{array}{l}\text { Coeficiente de amortigua- } \\
\text { miento (tan } \delta \text { ) }\end{array}$ & $\times 10^{-3}$ & 6,28 & 3,21 & 0,51 \\
\hline Coeficiente acústico $(K)$ & $\left(\mathrm{m}^{4} / \mathrm{s} \mathrm{kg}\right)$ & 4,80 & 0,355 & 0,07 \\
\hline
\end{tabular}
representada por la rigidez de la probeta $(E I)$ y la masa $(m)$ corresponde a la propia de la probeta, más la del acelerómetro. De acuerdo con la fórmula 8 , si la masa aumenta, la frecuencia disminuye, como es el caso del sistema formado por la probeta y el acelerómetro. De tal forma, que la masa del acelerómetro altera la frecuencia medida y en consecuencia el cálculo del módulo de elasticidad.

Transformando la fórmula 3 se obtiene la fórmula 3a:

$$
f_{n}=\sqrt{\frac{12,65 E_{v t} I}{m L^{3}}}
$$

Donde:

$E_{v t} \quad=$ módulo de elasticidad de la madera $(\mathrm{Pa})$

$f_{n} \quad=$ frecuencia natural de la probeta $(\mathrm{Hz})$

$m=$ masa de la probeta al momento del ensayo $(\mathrm{kg})$

$L \quad=$ longitud de la probeta $(\mathrm{m})$

I = momento de inercia de la sección transversal de la probeta $\left(\mathrm{m}^{4}\right)$.

Si en la ecuación 3 a se mantienen constantes el módulo de elasticidad $E_{v t}$ y las dimensiones de las probetas y en consecuencia el momento de inercia (I), la evolución de la frecuencia natural se puede modelar en función de la variación de la masa $(m)$ de la probeta. Introduciendo en la ecuación 3a los valores promedio a la sección trans- 
versal de las probetas y los valores de la tabla 1 correspondientes de $E_{v t}$ y $L$ para las probetas sin tratamiento, la figura 4 presenta la variación de la frecuencia natural de la probeta $\left(f_{n}\right)$ en función de su masa $(m)$. Esta tendencia está representada por la línea "Fórmula (3)". En la misma figura se muestra el valor de la frecuencia correspondiente a la masa promedio de las 30 probetas sin tratamiento (Promedio 30 probetas Q. scytophylla) y la frecuencia para una probeta con la masa del acelerómetro incorporada (Probeta con acelerómetro).

De la expresión para $f_{n}=f(m)$, presentada en la figura 4 , se deriva la ecuación 9, con la cual se puede estimar la frecuencia del sistema en función de su masa:

$$
f_{n}=224,47 m^{-0,5}
$$

Donde:

$$
\begin{array}{ll}
f_{n} & =\text { frecuencia }(\mathrm{Hz}) \\
m & =\text { masa }(\mathrm{kg})
\end{array}
$$

Igualmente, se puede emplear la fórmula 3 para graficar el módulo de elasticidad en función de la frecuencia, manteniendo constantes, esta vez, la masa y las dimensiones del sistema. La figura 5 presenta la variación del módulo de elasticidad en función de la frecuencia natural de las probetas. Esta tendencia está representada por la línea "Fórmula (3)". En la misma figura se muestra el módulo de elasticidad $E_{v t}$ en función del promedio de la masa de las 30 probetas sin tratamiento (Promedio 30 probetas Q. scytophylla) y el módulo de elasticidad $E_{v t}$ para una probeta con la masa del acelerómetro incorporada (Probeta con acelerómetro).

De la expresión para $E_{v t}=f\left(f_{n}\right)$, presentada en la figura 5 , se deriva la ecuación 10 , con la cual se puede estimar el módulo de elasticidad de una probeta en función de su frecuencia natural:

$$
E_{v t}=0,024 f_{n}^{2}
$$

Donde:

$E_{v t} \quad=$ módulo de elasticidad $(\mathrm{MPa})$

$f_{n} \quad=$ frecuencia natural $(\mathrm{Hz})$

La tabla 2 presenta, para las probetas sin y con el acelerómetro incorporado, la masa, la frecuencia natural y los módulos de elasticidad determinados de forma experimental. Además, se presentan las frecuencias y los módulos estimados con las fórmulas 9 y 10, así como las diferencias entre los valores experimentales y estimados. Estos valores conformaron las figuras 4 y 5 .

En la figura 4 se observa que la masa del sistema probeta-acelerómetro aumenta y la frecuencia disminuye. En

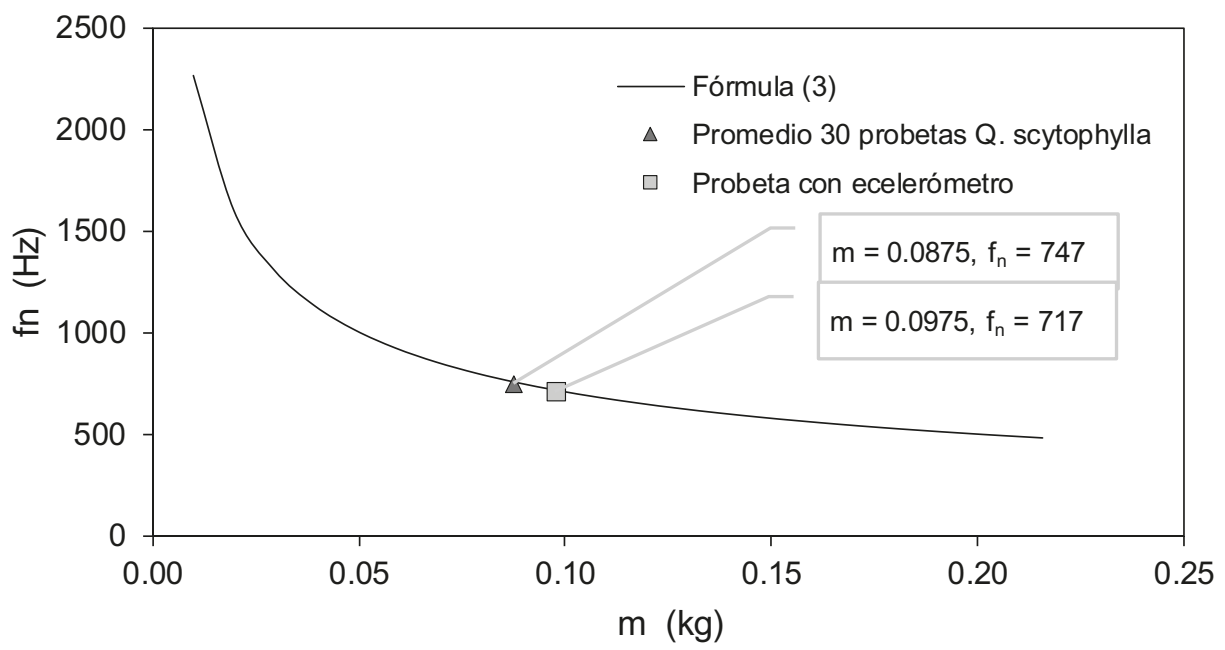

Figura 4. Variación de la frecuencia natural $\left(f_{n}\right)$ en función de la masa $(m)$ de la probeta sin y con acelerómetro. 


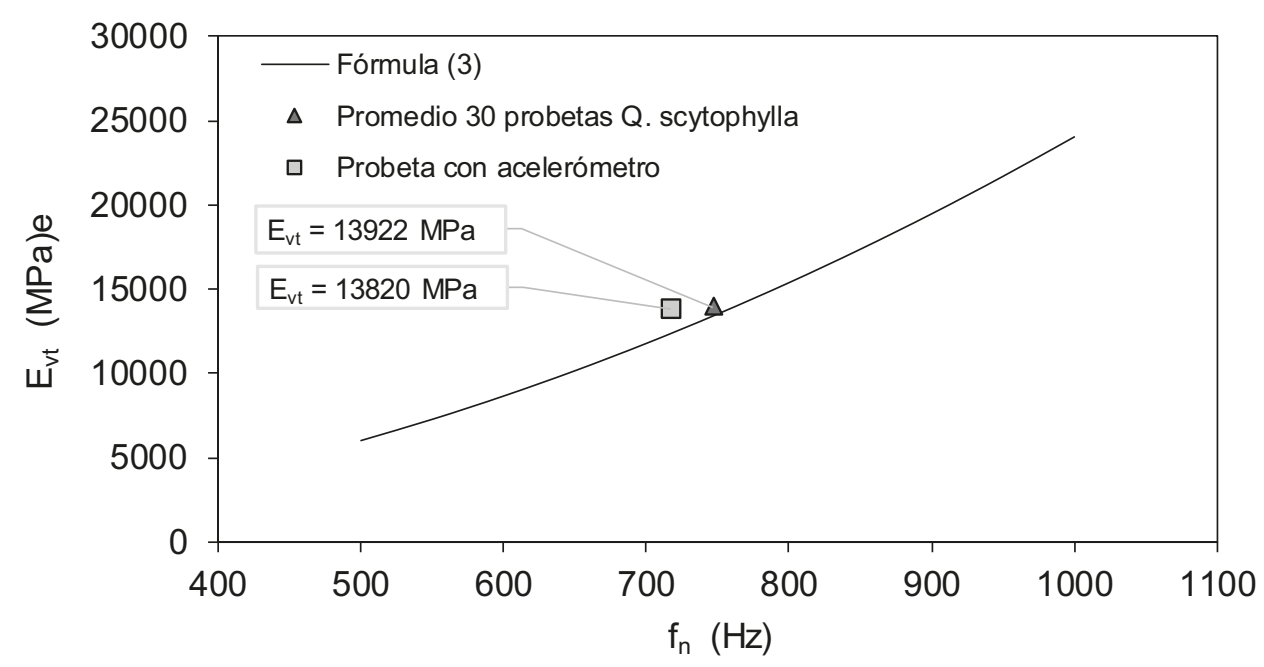

Figura 5. Variación del módulo de elasticidad $\left(E_{v t}\right)$ en función de la frecuencia natural $\left(f_{n}\right)$ de las probetas.

la figura 5 se observa que la frecuencia del sistema probeta-acelerómetro disminuye, pero el módulo de elasticidad es casi igual. La presencia del acelerómetro disminuyó la frecuencia $f_{n}$ en $4,01 \%$ respecto a la frecuencia del sistema sin considerar la masa del acelerómetro. Como consecuencia, el módulo de elasticidad también se vio afectado con una reducción de $0,73 \%$. Es decir, el valor promedio del módulo de elasticidad determinado en esta investigación está subvaluado.

Para verificar si la masa del acelerómetro influye estadísticamente en el valor promedio del módulo de elasticidad, la prueba de diferencia de medias entre los $E_{v t}$ calculados con la fórmula 3 , sin y con la masa del acelerómetro, demostró que no hay diferencia significativa entre las medias de las dos muestras con un nivel de confianza de $95 \%$. En efecto, de acuerdo con los resultados de ANOVA presentados en la tabla 3, y dado que el valor- $P$ calculado no es menor que 0,05 , no existe una diferencia estadísticamente significativa entre las medias de las 2 variables. De aquí sigue que, si bien la masa del acelerómetro influye en la medición de la frecuencia y modifica el cálculo del módulo de elasticidad, estadísticamente los resultados no son diferentes, argumento que permite validar la configuración de las pruebas de vibraciones.

\section{Características acústicas}

Los valores promedio y los intervalos de dispersión de los módulos de elasticidad, módulos específicos y coefi-

TABLA 2. Valores experimentales y estimados con las fórmulas 9 y 10.

\begin{tabular}{|c|c|c|c|c|c|c|c|}
\hline & $\begin{array}{c}m \\
(\mathrm{~kg})\end{array}$ & $\begin{array}{c}f_{n} \\
(\mathrm{~Hz})\end{array}$ & $\begin{array}{c}E_{v t} \\
(\mathrm{MPa})\end{array}$ & $\begin{array}{c}f_{n} \\
(\mathrm{~Hz})\end{array}$ & $\begin{array}{c}E_{v t} \\
(\mathrm{MPa})\end{array}$ & $\begin{array}{l}\Delta f_{n} \\
(\%)\end{array}$ & $\begin{array}{c}\Delta E_{v t} \\
(\%)\end{array}$ \\
\hline \multirow[t]{2}{*}{ Probeta } & \multicolumn{3}{|c|}{ Valor experimental } & \multicolumn{2}{|c|}{ Fórmula } & \multicolumn{2}{|c|}{ Valor estimado } \\
\hline & & & & 9 & 10 & & \\
\hline Sin acelerómetro & 0,0875 & 747 & 13922 & & 13820 & & $-0,73$ \\
\hline Con acelerómetro & 0,0980 & & & 717 & & $-4,01$ & \\
\hline
\end{tabular}

$m=$ masa; $f_{n}=$ Frecuencia natural; $E_{v t}=$ Módulo de elasticidad; $\Delta f_{n}=$ Variación en la frecuencia natural; $\Delta E_{v t}=$ Variación en el módulo de elasticidad 
TABla 3. Resultados de ANOVA.

\begin{tabular}{ccccc}
\hline Fuente & $\begin{array}{c}\text { Suma de } \\
\text { Cuadrados }\end{array}$ & $\begin{array}{c}\text { GL } \\
\text { Cuadrado } \\
\text { Medio }\end{array}$ & Valor-P \\
\hline Entre grupos & $4,18294 \times 10^{17}$ & 1 & $4,18294 \times 10^{17}$ & 0,6708 \\
Intra grupos & $1,32908 \times 10^{20}$ & 58 & $2,29151 \times 10^{18}$ & \\
Total & $1,33326 \times 10^{20}$ & 59 & & \\
\hline
\end{tabular}

$\mathrm{GL}=$ Grados de libertad; $P=$ Valor $P$ para un nivel de confianza de 95\%.

cientes de amortiguamiento presentados en la tabla 1, son similares a los exhibidos por Brémaud et al. (2011) para maderas con densidades $\rho$ que varían entre $800 \mathrm{~kg} /$ $\mathrm{m}^{3}$ y $1280 \mathrm{~kg} / \mathrm{m}^{3}$, con contenidos de humedad $\mathrm{CH}$ en promedio de $12 \%$ : módulos de elasticidad $E_{v t}$ entre 7200 MPa y $40960 \mathrm{MPa}$, módulos específicos $E_{\rho h}$ entre $9 \mathrm{~m}^{2} /$ $\mathrm{s}^{2}$ y $32 \mathrm{~m}^{2} / \mathrm{s}^{2}$ y coeficientes de amortiguamiento $\tan \delta$ entre $4,1 \times 10^{-3}$ y $15,2 \times 10^{-3}$.

Igualmente, Spycher et al. 2008 determinaron para madera de Picea abies con $\mathrm{CH}=10,5 \% ; \rho=360 \mathrm{~kg} / \mathrm{m}^{3}$ a $495 \mathrm{~kg} / \mathrm{m}^{3} ; E_{v t} 9979 \mathrm{MPa}$ a $14784 \mathrm{MPa} ;=1,7 \mathrm{~m}^{4} / \mathrm{s} \mathrm{kg}$ a $3,6 \mathrm{~m}^{4} / \mathrm{s} \mathrm{kg}$ y Acer pseudoplatanus con $\mathrm{CH}=10,5 \%, \rho=$ $569 \mathrm{~kg} / \mathrm{m}^{3}$ y $625 \mathrm{~kg} / \mathrm{m}^{3} ; E_{v t}=7315 \mathrm{MPa}$ y $10050 \mathrm{MPa} ; \mathrm{y}$ $\mathrm{K}$ de $2,3 \mathrm{~m}^{4} / \mathrm{s} \mathrm{kg}$ a $3 \mathrm{~m}^{4} / \mathrm{s} \mathrm{kg}$, resultados que son comparables a los obtenidos en esta investigación. En el mismo contexto, Roohnia et al. (2011a y 2011b) quienes puntualizaron para madera de Cupressus arizonica, con un contenido de humedad $\mathrm{CH}=10 \%$ y densidad promedio $\rho$ de $450 \mathrm{~kg} / \mathrm{m}^{3}$, el módulo de elasticidad $E_{v t}$ entre $3000 \mathrm{MPa}$ y $10000 \mathrm{MPa}$; el coeficiente de amortiguamiento $\tan \delta$ de $7 \times 10^{-3}$ a $16 \times 10^{-3}$; y el coeficiente acústico $K$ de $6,5 \mathrm{~m}^{4} / \mathrm{s}$ $\mathrm{kg}$ a $8,5 \mathrm{~m}^{4} / \mathrm{s} \mathrm{kg}$.

Igualmente, para maderas angiospermas con densidad similar a la de Q. scytophylla los resultados son del mismo orden que los de Traoré et al. (2010): $\rho_{b}$ entre 750 $\mathrm{kg} / \mathrm{m}^{3}$ y $800 \mathrm{~kg} / \mathrm{m}^{3} ; E_{v t}$ entre 13300 y $15200 ; E_{\rho b}$ entre $15,2 \mathrm{~m}^{2} / \mathrm{s}^{2}$ y $17 \mathrm{~m}^{2} / \mathrm{s}^{2} ; \tan \delta$ entre $7,5 \times 10^{-3}$ y $9,5 \times 10^{-3} ; \mathrm{y}$ $K$ entre $4,6 \mathrm{~m}^{4} / \mathrm{s} \mathrm{kg}$ y $6 \mathrm{~m}^{4} / \mathrm{s} \mathrm{kg}$, quienes utilizaron madera con un contenido de humedad de $12 \%$ y una configuración de los ensayos similar al de esta investigación.
Los resultados de la tabla 1 son comparables con los de maderas mexicanas del género Quercus estudiadas por Sotomayor-Castellanos y Suarez-Béjar (2013). Los autores determinaron, para probetas con dimensiones similares a las de esta investigación, sin y con tratamiento de higro-termo fatiga, con contenidos de humedad promedio de $12,6 \%$ y $13,2 \%$ y densidades promedio de $734 \mathrm{~kg} / \mathrm{m}^{3} \mathrm{y}$ $537 \mathrm{~kg} / \mathrm{m}^{3}$ respectivamente, frecuencias naturales en vibraciones transversales de $931 \mathrm{~Hz}$ y $903 \mathrm{~Hz}$, módulos de elasticidad entre 11805 y 11 200, módulos específicos entre $20,26 \mathrm{~m}^{2} / \mathrm{s}^{2}$ y $20,87 \mathrm{~m}^{2} / \mathrm{s}^{2}$, coeficientes de amortiguamiento de 7,67 y 7,65, y coeficientes acústicos de 6,06 $\left(\mathrm{m}^{4} / \mathrm{s} \mathrm{kg}\right)$ y $8,57\left(\mathrm{~m}^{4} / \mathrm{s} \mathrm{kg}\right)$.

De la misma manera, los resultados del $E_{v t}$ para $Q$. scytophylla, determinados en esta investigación, son comparables al módulo de elasticidad en vibraciones transversales en apoyos simple-simple determinado por Sotomayor-Castellanos et al. (2013) para madera de Quercus spp.

\section{Correlaciones}

Los valores promedio de densidades, frecuencias, módulos de elasticidad, módulos específicos y decrementos logarítmicos fueron parámetros determinados a partir de mediciones directas en laboratorio, es decir, son consideradas como variables de primer nivel. En contraste, los parámetros coeficiente de amortiguamiento y coeficiente acústico fueron derivados o cálculos de segundo nivel. De aquí, el análisis siguiente de correlaciones y pruebas $t$ de Student se circunscriben a las variables de primer nivel. La tabla 4 presenta los coeficientes de determinación $\left(R^{2}\right)$ para cada par de las variables: densidad $(\rho)$, frecuencia natural $\left(f_{n}\right)$, módulo de elasticidad en vibraciones transversales $\left(E_{v t}\right)$, módulo específico $\left(E_{\rho b}\right)$ y decremento logarítmico $(\delta)$, para los dos grupos de probetas PST y PCT.

De la observación de la tabla 4 , se deduce que las variables: $f_{n}$ PST y $f_{n}$ PCT correlacionan bien con $E_{v t}$ y $E_{\rho h}$ para PST y PCT, y que $E_{v t}$ PST y $E_{v t}$ PCT correlacionan bien con $E_{\rho h}$ PST y $E_{\rho h}$ PCT. Estas variables están implícitas en las ecuaciones empleadas para la determinación de los 
TAвla 4. Coeficientes de determinación $\left(R^{2}\right)$ entre variables.

\begin{tabular}{|c|c|c|c|c|c|c|c|c|c|}
\hline & $\rho_{h} \mathrm{PCT}$ & $f_{n}$ PST & $f_{n} \mathrm{PCT}$ & $E_{v t}$ PST & $E_{v t} \mathrm{PCT}$ & $E_{\rho h}$ PST & $E_{\rho h}$ PCT & $\delta$ PST & $\delta$ РСТ \\
\hline$\rho_{h}$ PST & 0,109 & 0,061 & $-0,070$ & 0,195 & $-0,080$ & 0,040 & $-0,125$ & $-0,058$ & $-0,080$ \\
\hline$\rho_{h}$ PCT & & $-0,444$ & 0,234 & $-0,276$ & 0,516 & $-0,301$ & 0,264 & $-0,235$ & $-0,226$ \\
\hline$f_{n}$ PST & & & $-0,257$ & $0,946^{*}$ & $-0,332$ & $0,954^{*}$ & $-0,231$ & 0,392 & 0,652 \\
\hline$f_{n}$ PCT & & & & $-0,266$ & $0,912^{*}$ & $-0,262$ & $0,955^{*}$ & $-0,221$ & $-0,160$ \\
\hline$E_{v t} \mathrm{PST}$ & & & & & $-0,295$ & $0,988^{*}$ & $-0,244$ & 0,375 & 0,623 \\
\hline$E_{v t} \mathrm{PCT}$ & & & & & & $-0,290$ & $0,962^{*}$ & $-0,272$ & $-0,055$ \\
\hline$E_{\rho h}$ PST & & & & & & & $-0,230$ & 0,394 & 0,646 \\
\hline$E_{\rho h}$ PCT & & & & & & & & $-0,230$ & 0,007 \\
\hline$\delta$ PST & & & & & & & & & 0,176 \\
\hline
\end{tabular}

parámetros de segundo nivel. En el mismo contexto, ninguna de las otras variables correlaciona bien entre ellas.

La tabla 5 presenta los resultados de las pruebas $t$ de Student para comparar las medias entre los grupos de probetas sin tratamiento $\left(\bar{x}_{s}\right)$ y con tratamiento $\left(\bar{x}_{c}\right)$ para la densidad $\left(\rho_{h}\right)$, la frecuencia $\left(f_{n}\right)$, el módulo de elasticidad $\left(E_{v t}\right)$, el módulo específico $\left(E_{\rho h}\right)$ y el decremento logarítmico $(\delta)$, Las pruebas consistieron en verificar la Hipótesis nula $H_{0}: \bar{x}_{s}=\bar{x}_{c}$ para un nivel de confianza de $95 \%$.

Para las pruebas de $\rho_{b}$ PST vs, $\rho_{b}$ PCT y de $\delta$ PST vs, $\delta$ PCT, el valor $P$ calculado es menor que 0,05 , en consecuencia, se puede rechazar la hipótesis nula, Además, el intervalo no contiene el valor de 0 , es decir, para nivel de confianza de $95 \%$, existe una diferencia significativa entre las medias de las dos muestras de datos para la densidad y el decremento logarítmico. Este resultado implica que el tratamiento higro-térmico modificó de manera significativa la densidad y el decremento logarítmico de la madera de Q, scytophylla.

En contraste, para las pruebas de $f_{n}$ PST vs, $f_{n}$ PCT, $E_{v t}$ PST vs, $E_{v t}$ PCT y de $E_{\rho H}$ PST vs, $E_{\rho H}$ PCT, el valor $P$ calculado no es menor que 0,05 , en consecuencia, no se puede rechazar la hipótesis nula, Además, el intervalo contiene el valor de 0 , es decir, para nivel de confianza de 95\%, no hay diferencia significativa entre las medias de las dos
TABLA 5. Valores $P$, intervalos y significancias de las pruebas $t$ de Student.

\begin{tabular}{cccc}
\hline \multicolumn{1}{c}{ Prueba } & $P$ & Intervalo & Significancia \\
\hline$\rho_{h}$ PST vs $\rho_{h}$ PCT & 0,0080595 & $\begin{array}{l}-0,188122, \\
-0,0294311\end{array}$ & $*$ \\
\hline \multirow{2}{*}{$f_{n}$ PST vs $f_{n}$ PCT } & 0,8911290 & $-22,1485$, & $* *$ \\
\hline & & 25,4152 & \\
$E_{v t}$ PST vs $E_{v t}$ PCT & 0,7217690 & $-786,808$, & $* *$ \\
\hline & & 1129,34 & \\
$E_{\rho h}$ PST vs $E_{\rho h}$ PCT & 0,9944050 & $-1,04587$, & $*$ \\
\hline & & 1,03854 & \\
$\delta$ PST vs $\delta$ PCT & 0,0080595 & $-0,188122$, & $*$ \\
\hline
\end{tabular}

$\rho_{h}=$ Densidad $;=$ Frecuencia natural $; E_{k t}=$ Módulo de elasticidad $; E_{\rho h}=$ Módulo especíico; $\delta$ = Decremento logarítmico; PST = Probetas sin tratamiento; PCT = Probetas con tratamiento; $P=$ Valor $P$ para un nivel de confianza de $95 \%$. * Sí existe diferencia significativa para nivel de confianza de $95 \%(P<0,001)$

** No existe diferencia significativa para nivel de confianza de $95 \%$.

muestras de datos, Este resultado sugiere que el tratamiento higro-térmico no modificó de manera significativa estos parámetros.

La densidad y el coeficiente de amortiguamiento fueron afectados por el tratamiento, resultados que coin- 

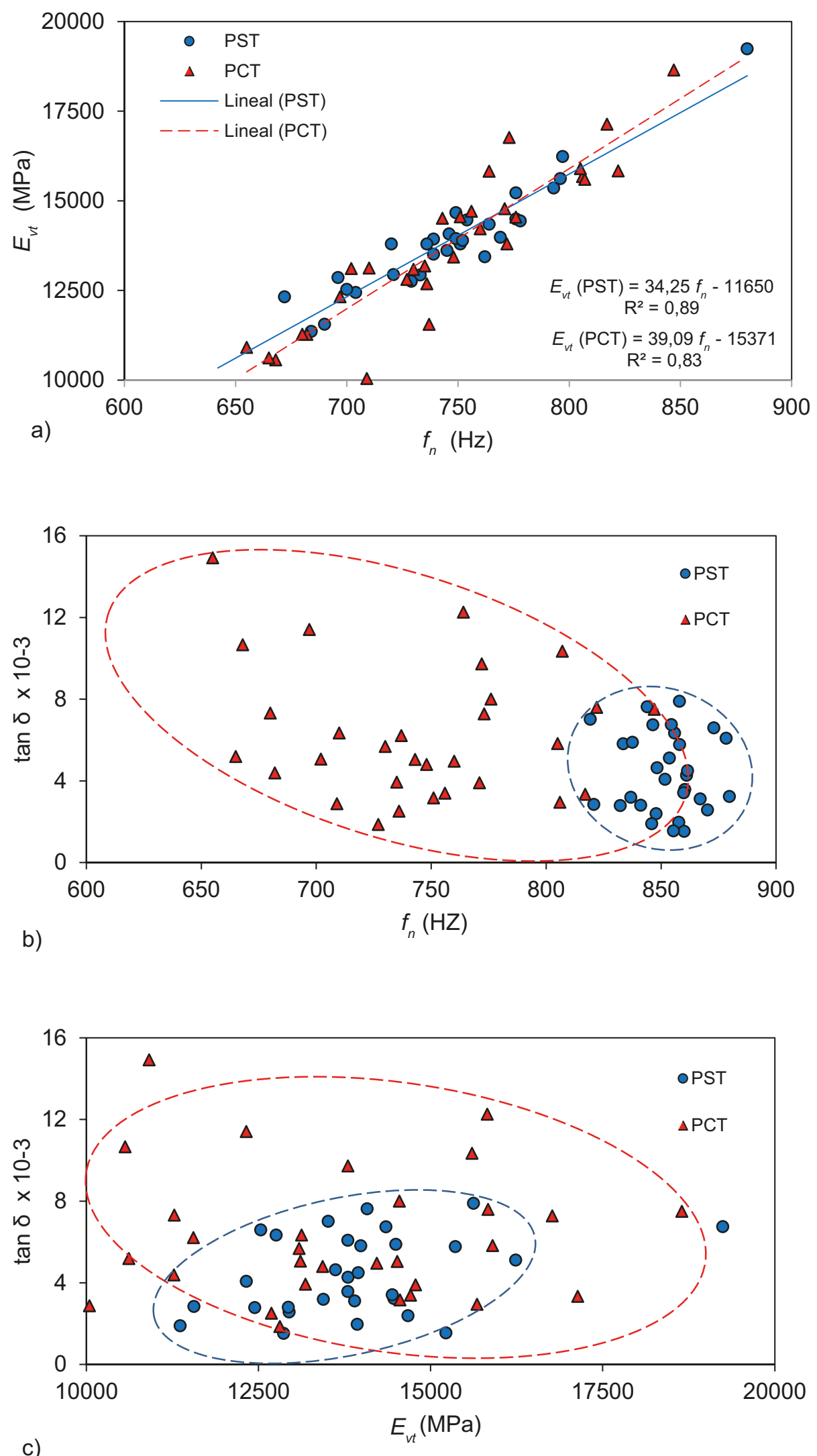

Figura 6. Variación de $f_{n}, E_{v t}$ y tan $\delta$ al interior de la especie Q. scytophylla. 

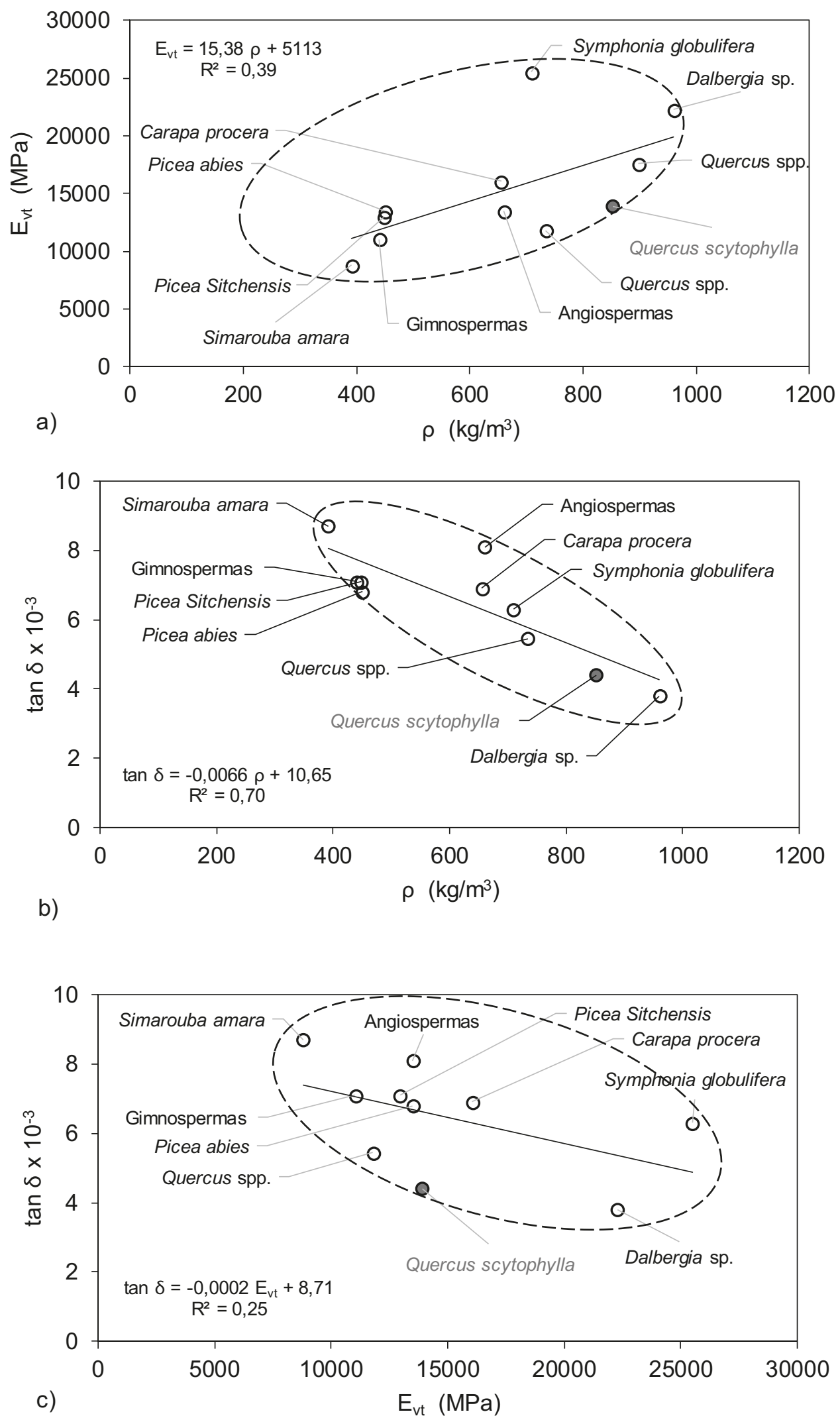

Figura 7. Variación de $f_{n}, E_{v t}$ y $\tan \delta$ entre especies y Q. scytophylla . 
ciden con los de Korkut et al. (2010), y en cambio, el tratamiento no afecto de manera estadísticamente significativa, la frecuencia y los módulos de elasticidad y específico.

\section{Variación al interior de la especie $Q$. scytophylla}

El módulo de elasticidad $E_{v t}$ aumenta proporcionalmente y correlaciona bien con la frecuencia $f_{n}$ para los dos grupos de madera (Fig. 6a) coincidiendo con los resultados de Obataya et al. (1998) y Spycher et al. (2008). En contraste, el coeficiente de amortiguamiento $\tan \delta$ es independiente de la frecuencia (Fig. 6b), pero se observa una ligera tendencia para las probetas con tratamiento (PCT) de la disminución del coeficiente $\tan \delta$ cuando la frecuencia decrece, coincidiendo con los resultados de Obataya et al. (1998) y Spycher et al. (2008). Estos resultados confirman lo propuesto por Ouis (2003) quien opina que las propiedades elásticas y de amortiguamiento de la madera están relacionadas con la frecuencia. Sin embargo, coincidiendo con los resultados de (Roohnia et al. 2011a y 2011b), ambas variables son independientes entre si (Fig. 6c).

\section{Variación entre especies}

Comparativamente con los valores del módulo de elasticidad $E_{v t}$ de otras especies, la magnitud del módulo de $Q$. scytophylla es proporcional a su densidad $\rho$, de manera similar que otras maderas (Fig. 7a). Los datos de las especies presentados en la figura 7 provienen de las referencias citadas en la sección de la introducción. De los resultados anteriores, se deduce que el coeficiente de amortiguamiento $\tan \delta$ disminuye a medida que $\rho$ decrece (Fig. 7b) y disminuye en función de $E_{v t}$ (Fig 7c) coincidiendo con Brémaud et al. (2012),

\section{CONCLUSIONES}

Se estudió el efecto del tratamiento higro-térmico en las características acústicas de la madera de Quercus scytophylla. La investigación demuestra que el tratamiento no modifica el módulo de elasticidad en vibraciones transversales y el módulo específico. Sin embargo, el tratamiento altera de manera estadísticamente significativa la densidad y el decremento logarítmico de la madera de Q. scytophylla. Para la madera sin tratamiento y con tratamiento, la frecuencia natural correlaciona con los módulos de elasticidad y específicos. En contraste, el decremento logarítmico y el coeficiente de amortiguamiento no correlacionan bien entre ellos. La presencia del acelerómetro empleado para medir el movimiento de las probetas, influye en los cálculos, de tal forma que la frecuencia disminuye y en consecuencia el valor del módulo de elasticidad calculado es subestimado. La magnitud de la variación de los resultados entre las probetas ensayadas, es del mismo orden que la propuesta por otros autores para maderas con características tecnológicas similares a la de Q. Scytophylla. Igualmente, los resultados para Q. scytophyla son similares a las características de otras maderas

\section{AGRADECIMIENTOS}

Al Dr, Ying Hei Chui Profesor de la Universidad de New Brunswick de Canadá, por facilitar el equipo para las pruebas de vibraciones. Al M.C. Saúl Antonio Hernández Maldonado estudiante de Doctorado en la Universidad de New Brunswick de Canadá, por la capacitación en el uso del equipo de vibraciones. A la Coordinación de la Investigación Científica de la Universidad Michoacana de San Nicolás de Hidalgo, Morelia, Michoacán, México.

\section{REFERENCIAS}

Aramaki, M. 2007. Sound quality assessment of wood for xylophone bars. Journal of the Acoustical Society of America 121(4):2407-2420.

Arizaga, S., J. Martínez C., M. Salcedo C. y M.A. Bello G. 2009. Manual de la biodiversidad de encinos michoacanos. Secretaría de Medio Ambiente y Recursos Naturales. Instituto Nacional de Ecología. México. 147 p.

Ashby, M.F. 2010. Materials selection in mechanical design. $4^{\text {th }}$ edition. Elsevier. USA. 664 p.

Barajas M., J. y C. León G. 1984. Anatomía de maderas de México: Especies de una selva caducifolia. Instituto de Biología. Publicaciones especiales 1. Universidad Nacional Autónoma de México. 163 p. 
Bello G., M.A. y J.N. Labat. 1987. Los encinos (Quercus) del Estado de Michoacán, México. Collection Etudes Mesoaméricaines. Centre d’Etudes Mexicaines et Centraméricaines. Secretaría de Agricultura y Recursos Hidráulicos. Instituto Nacional de Investigaciones Forestales y Agropecuarias. México. 93 p.

Bodig, J. y B.A. Jayne. 1982. Mechanics of wood composites. Van Nostrand Reinhold Company. EUA. 711 p.

Brancheriau, L., H. Baillères, P. Détienne, J. Gril y R. Kronland. 2006a. Key signal and wood anatomy parameters related to the acoustic quality of wood for xylophone-type percussion instruments. Journal of Wood Science 52:270273.

Brancheriau, L., H. Baillères, P. Détienne, R. Kronland y B. Metzger. 2006b. Classifying xylophone bar materials by perceptual, signal processing and wood anatomy analysis. Annales of Forest Sciences 63:73-81.

Brancheriau, L., C. Kouchade y I. Brémaud. 2010. Internal friction measurement of tropical species by various acoustic methods. Journal of Wood Science 56:371-379.

Brémaud, I., Y. El Kaïm, D. Guibal, K. Minato, T. Thibaut y J. Gril. 2012. Characterization and categorization of the diversity in viscoelastic vibrational properties between 98 wood types. Annals of Forest Science 69:373-386.

Brémaud, I., J. Gril y B. Thibaut. 2011. Anisotropy of wood vibrational properties: dependence on grain angle and review of literature data. Wood Science and Technology 45:735-754.

Brémaud, I., K. Minato, P. Langbour y B. Thibaut. 2010. Physico-chemical indicators of inter-specific variability in vibration damping of wood. Annals of Forest Sciences 67(7):707, p:1-8

Bucur, V. 2006. Acoustics of wood. 2a ed. Springer series in wood. Springer Berlin Heidelberg. Alemania. 393 p.

De la Paz Pérez O., C., Vélez J., S. y J. Ceja R. 2006. Anatomía de la madera de ocho especies de Quercus (Fagaceae) de Oaxaca, México. Madera y Bosques 12(1):63-94.

De la Paz Pérez O., C., S. Vélez J., A. Serrano J. y B. Rochin G. 2002. La madera de las guitarras de Paracho, Michoacán. ContactoS 44:45-53.
Estevez, B.M. y H.M. Pereira. 2009. Wood Modification by Heat Treatment: A Review. Bioresources 4(1):370-404.

Guridi G., L.I. y A. García L. 1997. Las maderas en los instrumentos musicales de cuerda de Paracho. Secretaría de Difusión cultural. Universidad Michoacana de San Nicolás de Hidalgo. México. 45 p.

Gutiérrez C., L. y J. Dorantes L. 2007. Especies forestales de uso tradicional del Estado de Veracruz. Conafor-Conacyt-UV 2003-2004. México. Consultado julio 2014. Disponible en: http://www.verarboles.com/.

Gutiérrez P., H. y R. de la Vara S. 2012. 3a Edición. Análisis y diseño de experimentos. Mc Graw Hill. México. 489 p.

International Organization for Standardization. 2012. ISO 3129:2012 (E). Wood - Sampling methods and general requirements for physical and mechanical tests. ISO Cata$\log 79$ Wood technology; 79.040 Wood, sawlogs and saw timber. International Organization for Standardization. Brussels.

Kawamoto, S. y R.S. Williams. 2002. Acoustic emission and acousto-ultrasonic techniques for wood and wood-based composites - A review. Gen. Tech. Rep. FPL-GRT-134. U.S. Department of Agriculture. Forest Service. Forest Products Laboratory. USA. 16 p.

Korkut, S., S. Karayilmazlar, S. Hiziroglu y T. Sanli. 2010. Some of the properties of heat-treated sessile oak (Quercus petraea). Forest Products Journal 60(5):473-480.

Meyers, M.A. 1994. Dynamic behavior of materials. John Wiley \& Sons. USA. 668 p.

Minato, K., Y. Konaka, I. Bremaud, S. Suzuki y E. Obataya. 2010. Extractives of muirapiranga (Brosimum sp.) and its effects on the vibrational properties of wood. Journal of Wood Science 56:41-46.

Obataya, E., M. Norimoto y J. Gril. 1998. The effects of adsorbed water on dynamic mechanical properties of wood. Polymer 39(14):3059-3064.

Olguín-Cerón, J.B. y J.R. Sotomayor-Castellanos. 2013. Plastificado higro-térmico de madera de Quercus scytophylla. Investigación y Ciencia 59:25-33.

Oltean, L., A. Teischinger y C. Hansmann. 2007. Influence of temperature on cracking and mechanical properties of 
wood during wood drying - A review. BioResources 2(4):789-811.

Orduña B., F. y A. Quintanar I. 1992. Letter to the Editor: A preliminary determination of the mechanical properties of four species of tropical wood from Mexico. Journal of Sound and Vibration 154(2):365-368.

Ortiz M., R., E. Baradit, A. y J. Navarrete A. 2009. Estudio del efecto de la dirección de medición y contenido de humedad en la velocidad de ultrasonido en madera de Pinus radiata $\mathrm{D}$. Don a través de análisis de varianza. Ingeniería e Investigación 29(3):139-141.

Ouis, D. 2003. Effect of structural defects on the strength and damping properties of a solid material. European Journal of Mechanics A/Solids (22): 47-54.

Pellerin. R.F. y R.J. Ross. 2002. Nondestructive Evaluation of Wood. Forest Products Society. USA. 210 p.

Quintanar I., A., M. de Icaza H., L. Rivera N. y C. de la Paz Pérez O. 1998. Algunas características anatómicas y acústicas de tres especies de angiospermas de Huayacocotla, Ver. Madera y Bosques 4(1):15-25.

Raichel, D.R. 2006. The science and applications of acoustics. 2a ed. Springer. EUA. 660 p.

Roohnia, M., M. Hossein, S. Alavi-Tabar, A. Tajdini, A. JahanLatibari y N. Manouchehri. 2011a. Acoustic properties in Arizona cypress logs: a tool to select wood for sounding board. BioResources 6(1):386-399.

Roohnia, M., A. Tajdini y N. Manouchehri. 2011b. Assessing wood in sounding boards considering the ratio of acoustical anisotropy. NDT\&E International 44:13-20.

Sandoz, J.L., Y. Benoit y L. Demay. 2000. Wood testing using acousto-ultrasonic. In: Proceedings of the WCTE 2000 World Conference on Timber Engineering. Canada. p:136-142.

Sedik, Y., S. Hamdan, I. Jusoh y M. Hasan. 2010. Acoustic properties of selected tropical wood species. Journal of Nondestructive Evaluation 29:38-42.

Shen, J. 2006. Relationships between longitudinal and radial Picea genera sound vibration parameters. Frontiers of Forestry in China 1(4): 431-437.

Silva G., J.A., F.J. Fuentes T., R. Rodríguez A., P.A. Torres A., M.G. Lomelí R., C. Waitkus, y H.G. Richter. 2010. Fichas de propiedades tecnológicas y usos de maderas nativas de México e importadas. Departamento de Madera, Celulosa y Papel, Universidad de Guadalajara y Comisión Nacional Forestal. México. 207 p.

Sotomayor-Castellanos, J.R., Y. Banda-Cervantes, M. Ramírez-Pérez y G. Suárez-Béjar. 2013. Características dinámicas e indicadores de calidad de la madera de Quercus spp. (encino) del estado de Michoacán estudiada por métodos no destructivos. Investigación e Ingeniería de la Madera 9(2):14-29.

Sotomayor-Castellanos, J.R., L.I. Guridi G. y T. García M. 2010. Características acústicas de la madera de 152 especies mexicanas. Velocidad del ultrasonido, módulo de elasticidad, índice material y factor de calidad. Base de datos. Investigación e Ingeniería de la Madera 6(1):3-32. Sotomayor-Castellanos, J.R., M. Ramírez-Pérez y J.B. OlguínCerón. 2014. Caracterización en flexión estática de madera plastificada de Quercus scytophylla. Ciencia Nicolaita 62:17-33.

Sotomayor-Castellanos, J.R. y G. Suárez-Béjar. 2013. Efecto del tratamiento de higro-térmo-fatiga en las características acústicas de la madera de Pinus douglasiana y Quercus spp. Ciencia Nicolaíta 59:7-20.

Sotomayor-Castellanos, J.R. y J.B. Olguín-Cerón. 2014. Caracterización mecánica por ondas de esfuerzo de madera plastificada de Quercus scytophylla. Investigación y Ciencia 61:34-42.

Spycher, M., F.W.M.R. Schwarze y R. Steiger. 2008. Assessment of resonance wood quality by comparing its physical and histological properties. Wood Science and Technology 42: $325-342$.

Tamarit U., J.C. y J.L. López T. 2007. Xilotecnología de los principales árboles tropicales de México. Libro Técnico No. 3. Instituto Nacional de Investigaciones Forestales, Agrícolas y Pecuarias. México. 261 p.

Traoré, B., L., Brancheriau, P. Perré, T. Stevanovic y P. Diouf. 2010. Acoustic quality of vène wood (Pterocarpus erinaceus Poir.) for xylophone instrument manufacture in Mali. Annals of Forest Sciences 67:815, pp 1-7.

Wegst, U.G.K. 2006. Wood for sound. American Journal of Botany 93(10):1439-48. 
Wegst, U.G.K. 2008. Bamboo and wood in musical instruments. Annual Review of Materials Research 38: 323349.

Zhang, T., S-L. Bai, S. Bardet, T. Alméras, B. Thibaut y J. Beauchêne. 2011. Radial variations of vibrational properties of three tropical Woods. Journal of Wood Science 57:377-386.
Manuscrito recibido el 7 de mayo de 2013.

Aceptado el 28 de agosto de 2014

Este documento se debe citar como:

Sotomayor-Castellanos, J.R., G. Suárez-Béjar y J.B. Olguín-Cerón. 2015. Efecto del tratamiento higro-térmico en las características acústicas de la madera de Quercus scytophylla Liebm. Madera y Bosques 21(1):139-156. 